\title{
Enhancing the Physiology and Effectiveness of Whole-Body Cryotherapy Treatment for Sports Recovery by Establishing an Optimum Protocol: A Review of Recent Perspectives
}

\author{
Adnan $\mathrm{Haq}^{1^{*}}$, William J Ribbans ${ }^{2}$ and Anthony W Baross ${ }^{3}$ \\ ${ }^{1}$ Moulton College, UK \\ ${ }^{2}$ The University of Northampton, UK \\ ${ }^{3}$ University of Northampton Park Campus, UK
}

\begin{abstract}
Whilst several modalities have been adopted to promote optimum sports recovery, the beneficial effects of WholeBody Cryotherapy (WBC) remain equivocal. Physiological and performance effects of WBC treatment covering antiinflammatory, cardiovascular and autonomic changes as well as muscle damage marker reductions are well documented. However, evidence concerning the implications of manipulating WBC protocol factors are relatively scant and were the subject of this review. We attempted to address the question as to what is considered the optimum WBC protocol for post exercise recovery. Notable factors were identified as pertinent to the potential efficacy of WBC treatment. Firstly, treatment timing appears to influence WBC effectiveness, probably due to the time course of the inflammatory response post exercise. This can have direct applications for sports practice since many athletes lack immediate access to cryogenic chambers. It is probable that applying WBC within 60 minutes is desirable. Secondly, inter-individual factors such as body fat, age and sex affect WBC response, with young and lean males benefiting apparently more from the treatment. Furthermore, adjustments in WBC temperature, duration and frequency are to be factored in, with the latter potentially being particularly significant. The precise prescription of WBC treatment for optimising recovery could be affected accordingly. Understanding how different WBC protocols can ameliorate the effects of muscle damage can aid practitioners in the application of WBC strategies to facilitate recovery needs and athletic performance. Future studies should consider randomised controlled trials addressing the impact of protocol factors in isolation on physiological and performance parameters.
\end{abstract}

\section{Keywords}

Whole Body Cryotherapy/Cryostimulation, Recovery, Muscle damage, Performance

\section{Abbreviations}

CK: Creatine Kinase; CWI: Cold Water Immersion; DOMS: Delayed-Onset Muscle Soreness; EIMD: Exercise-Induced Muscle Damage; NIRS: Near Infra-Red Spectroscopy; sICAM-1: Soluble Intercellular Adhesion Molecule-1; WBC: Whole Body Cryotherapy

\section{Introduction}

Athletes place considerable demands on their bodies when undergoing training programmes to facilitate competition performance. Of notable concern is exercise recovery, the ability to restore the body to the pre-workout state, encompassing the restoration of physiological disturbances induced by exercise. Such disturbances might include glycogen depletion, hyperthermia, disruption of muscle fibres (including $\mathrm{Ca}^{2+}$ perturbations) and/or accumulation of lactate [1]. Accelerated recovery following strenuous exercise enables athletes to perform subsequent quality training sessions by taking full advantage of training stimuli and adaptations to improve skill and performance [1]. Owing to an advanced understanding of sport science, recovery practice has become more sophisticated in recent decades. Several methods of post-exercise recovery have been advocated,

*Corresponding author: Adnan Haq, Associate Lecturer/PhD Student, Moulton College, West Street, Moulton, Northamptonshire, NN3 7RR, UK, Tel: 01604-491131

Accepted: November 26, 2018

Published online: November 28, 2018

Citation: Haq A, Ribbans WJ, Baross AW (2018) Enhancing the Physiology and Effectiveness of Whole-Body Cryotherapy Treatment for Sports Recovery by Establishing an Optimum Protocol: A Review of Recent Perspectives. J Phys Med 1(1):41-52 


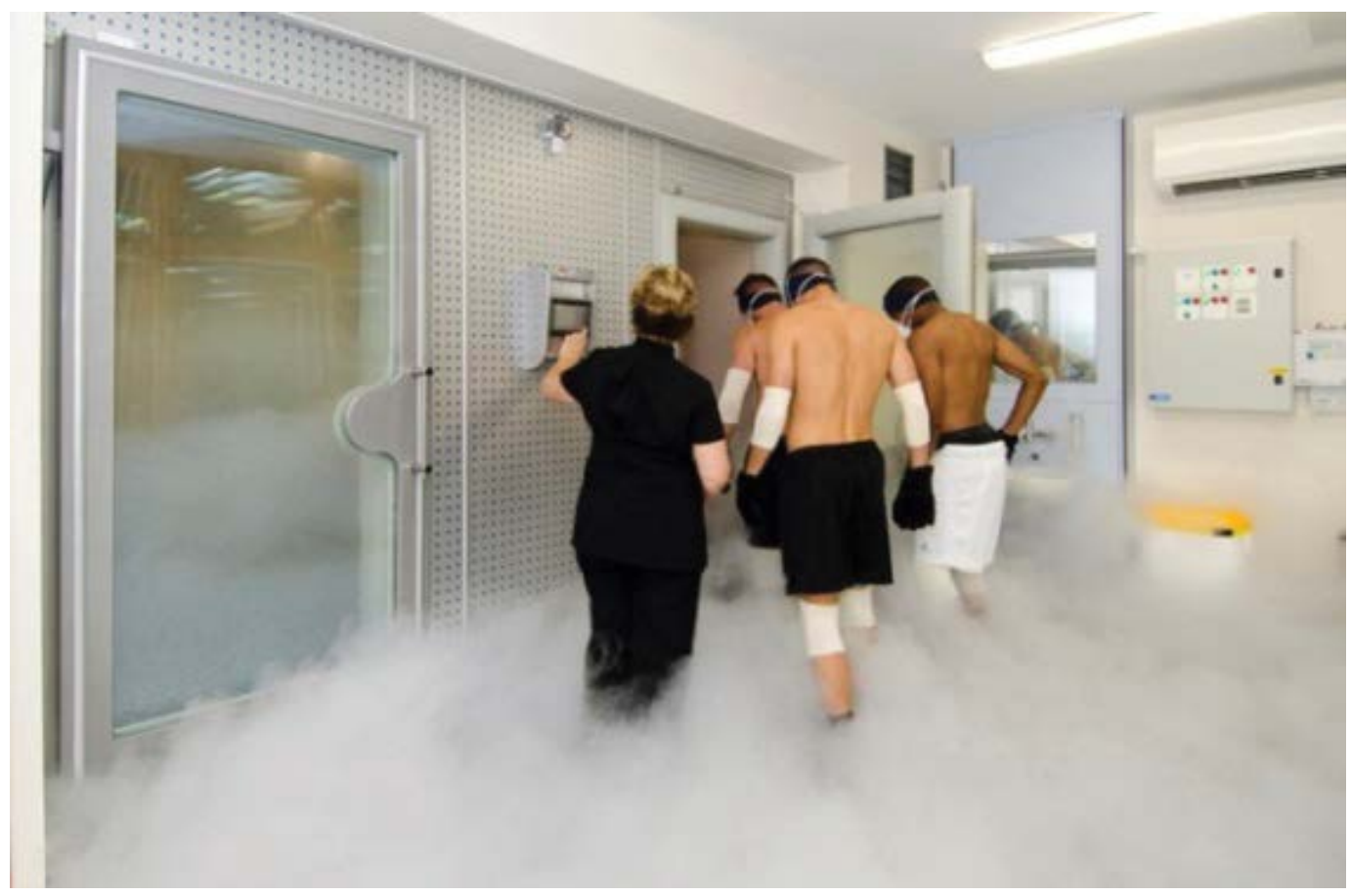

Figure 1: An example of a whole-body cryogenic chamber treatment (Chris Moody Rehabilitation Centre, Moulton, Northamptonshire), typically utilising liquid nitrogen as the coolant, with temperatures set at $-110^{\circ} \mathrm{C}$ to $-140^{\circ} \mathrm{C}$.

notably hydration, nutrition, electrostimulation, stretching, sleep and massage $[2,3]$. One emerging recovery tool is cryotherapy which involves cold temperatures to reduce swelling and soreness. Sports personnel are familiar with the practice of ice baths or cold-water immersions (CWI), the use of which has been extensively reviewed [4,5]. However, the recent emergence of the extreme cold present in whole body cryotherapy chambers or 'cryosaunas' has added an additional tool to sports recovery practice.

Whole body cryotherapy (WBC) was first introduced in Japan [6] and involves exposure to cold dry cryogenic gas, (e.g. liquid nitrogen) typically below $-100{ }^{\circ} \mathrm{C}$. WBC has been shown to mitigate the symptoms of musculoskeletal conditions, including ankylosing spondylitis [7], adhesive capsulitis [8] and fibromyalgia [9], demonstrating influential effects on soft tissue injury repair. The clinical benefits are principally associated with the anti-inflammatory effect of cryotherapy as well as its pain reduction properties, which are potential mechanisms by which sports recovery can be enhanced. Participants are usually exposed to extreme cold air temperatures in two parts: a vestibule chamber in temperatures between $-40{ }^{\circ} \mathrm{C}$ to $-60{ }^{\circ} \mathrm{C}$ for under a minute, followed by the main chamber where temperatures range from $-100{ }^{\circ} \mathrm{C}$ to $-140{ }^{\circ} \mathrm{C}[10,11]$ (Figure 1 ).

Whilst comprehensive WBC reviews are available [1214], there is a lack of discussion concerning what might be considered the optimum WBC protocol, along with implications for physiology and performance. This is potentially important for sports practitioners by informing them on how to optimise treatments for maximum impact. The main purpose of this review was to examine the literature covering the physiological and performance effects of WBC treatment for post exercise recovery and evaluate factors that could influence the potency of WBC to propose a potentially optimum protocol.

\section{Methods}

A computerised literature search was conducted on PubMed, Google Scholar and Science Direct. Initially, the following key terms were searched for: 'Whole Body Cryotherapy' or 'Whole Body Cryostimulation' or 'Partial Body Cryotherapy' with the field specified as 'title/abstract'. Resulting papers were scrutinised according to their potential relevance in addressing the themes of post exercise recovery and optimum protocols. Papers were rejected if they were conference proceedings, abstracts were not available, human subjects were not used or the article was not in English. With the remaining papers, a further search was performed to include the terms 'recovery' or 'sport' or 'exercise' or 'inflammation/inflammatory' with the field specified as 'title/ abstract'. Inclusion criteria for eligibility of review was then as follows: 1) Randomised controlled trial involving more than one group of participants or more than one condition; 2) Measurement of at least one physiological parameter (e.g. skin temperature, plasma IL-6); 3) Use of whole or partial body cryotherapy treatment with temperatures below -80 ${ }^{\circ} \mathrm{C}$ (the highest temperature used in the literature was -85 ${ }^{\circ} \mathrm{C}$ whilst all other studies used temperatures below -100 ${ }^{\circ} \mathrm{C}$ ). Only original research articles were considered at this stage. Reference lists of articles were examined for further identification of relevant studies.

\section{Results}

65 articles were revealed following the two literature searches including the key terms specified earlier. The majority of these were rejected on the premise that the meth- 


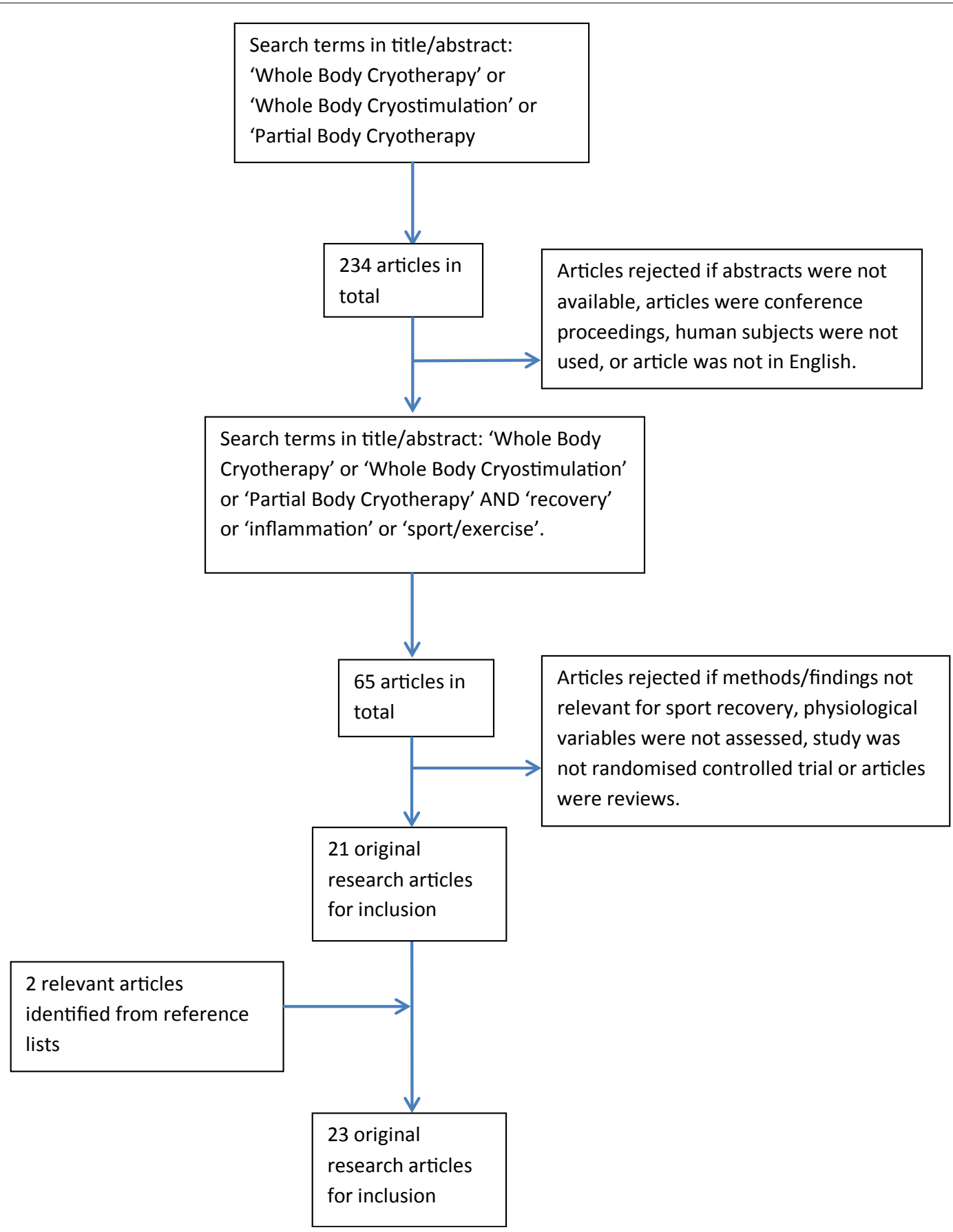

Figure 2: Identification and selection of research articles meeting specific criteria through literature search strategy, covering physiological and performance effects of WBC.

odologies and/or findings were not relevant for post exercise recovery (e.g. studies on ankylosing spondylitis, multiple sclerosis etc.), physiological variables were not assessed, study was not a randomised controlled trial or articles were not original research papers. Subsequently, 21 research articles were identified that met the specified criteria. A further two articles were identified $[11,15]$ from reference lists and were deemed appropriate additions for inclusion as they examined the response to whole body cryotherapy treatments post exercise. Figure 2 summarises the literature search protocol for research articles. Additionally, 16 research studies, 22 review articles and 2 textbook chapters that did not satisfy the above criteria have been referenced, including citations in the in- troduction. The additional references allow expansion of the review to consider relevant issues, including muscle damage, cold water immersion therapy and WBC protocol manipulation.

\section{Discussion}

\section{Overview of physiological effects of WBC}

WBC temperatures are significantly colder than humans have become habituated to. Upon exposure to such temperatures, cutaneous thermoreceptors are stimulated, and the brain's hypothalamus registers a potentially lifethreatening environment. With extreme cold effects being 
Citation: Haq A, Ribbans WJ, Baross AW (2018) Enhancing the Physiology and Effectiveness of Whole-Body Cryotherapy Treatment for Sports Recovery by Establishing an Optimum Protocol: A Review of Recent Perspectives. J Phys Med 1(1):41-52

well established [16], scientists and sports practitioners can seek to harness the physiological threat that extreme cold poses to the human body. Some physiological effects of WBC are outlined, alongside potential considerations for optimum WBC protocol factors.

\section{Skin temperatures and blood flow}

The impact of cryotherapy on skin temperatures and blood flow indicates the heat exchange occurring between the internal tissues and external environment [17]. Costello, et al. [18] utilised $-110^{\circ} \mathrm{C}$ WBC treatments lasting 3 minutes, 40 seconds and reported an average reduction of $12.1{ }^{\circ} \mathrm{C}$ in thigh skin temperatures immediately post WBC, followed by rapid recovery towards baseline levels within 60 minutes. This skin temperature ' $U$ ' response can be attributed to the 'rebound' effect with initial immediate cold induced vasoconstriction followed by vasodilation following removal from cold exposure, with consequent increase in blood flow and heat conductance from deep to superficial layers [19]. This hyperaemic effect is theorised to promote recovery through the removal of waste products, as well as enhancing oxygen delivery to hypoxic tissues. Using infrared thermal imaging on lower limbs, Hausswirth, et al. [20] reported an average skin temperature drop of $13.7^{\circ} \mathrm{C}$ following 3-minute WBC exposures at $-110{ }^{\circ} \mathrm{C}$. This could have implications for pain perception, since it exceeds the $13.6^{\circ} \mathrm{C}$ threshold required to obtain an analgesic effect [21]. Due to the 'rebound' effect, the skin temperature would likely return above the threshold following removal from the chamber. Clearly, treatment duration and temperature are to be factored in with regards to potential skin temperature decrements and how prolonged this effect lasts. The majority of WBC studies utilised treatments for a duration of 3 minutes with temperatures varying from $-110{ }^{\circ} \mathrm{C}$ to $-140{ }^{\circ} \mathrm{C}$. Whilst Hausswirth, et al. [20] reported significant skin temperature reductions at the higher end of this temperature range, it is conceivable that colder treatments will result in more pronounced changes to skin temperature. However, there is no experimental evidence to support this theory. The only study to have investigated the direct impact of differing WBC treatment durations on physiological variables was conducted by Selfe, et al. [10] who unsurprisingly found that 3-minute durations resulted in more significant skin temperature reductions than 1- and 2-minute treatments. In addition, the colder treatments used in this study $\left(-135^{\circ} \mathrm{C}\right)$ did not cause any lower skin temperatures compared to the aforementioned studies, suggesting that adjusting treatment temperature might not be a significant factor in achieving optimum skin temperature reductions.

To conclude, it would appear that 3-minute WBC durations at temperatures from $-110-140{ }^{\circ} \mathrm{C}$ are appropriate for achieving desired skin temperature reductions, with significantly longer durations considered unnecessary and potentially unsafe for the majority of practitioners.

\section{Muscle metabolism, function and damage}

Temperature-induced reductions in blood flow to muscles can contribute to reductions in muscle metabolism and temperature, potentially curtailing further muscular damage and swelling [17], as well as ischaemia-induced secondary cell injury [22]. Using near infra-red spectroscopy (NIRS) to measure muscle oxygenation, one study has experimentally demonstrated that WBC can blunt muscle metabolism [10]. However, it should be noted that NIRS does not permit direct measurement of muscle 'metabolism', rather only muscle oxygenation. Moreover, Selfe, et al. [10] applied WBC the day after exercise, which may be too late for WBC to have its optimal desired effect. These points make it difficult to draw tenable conclusions regarding the impact of WBC on muscle metabolism during the post-exercise recovery period. It would be reasonable to hypothesise that colder and longer WBC treatments would result in more pronounced muscle temperature decreases and potentially muscle blood flow. Two studies have demonstrated that WBC can reduce deep muscle tissue temperatures $[23,24]$. The former study used 3 minute 40 second treatments at $-110{ }^{\circ} \mathrm{C}$ whereas the latter only used 2 minutes at the same temperature. The shorter treatment appeared to result in comparable muscle temperature reductions to the longer treatment in the earlier study. Moreover, there is lack of evidence to substantiate the significance of this response for enhancing sports recovery and the optimal decrease in muscle temperature to benefit recovery has not been established.

Exercise-induced muscle damage (EIMD) commonly occurs following strenuous exercise, particularly if unaccustomed or involving predominantly eccentric contractions [25]. The aetiology and mechanisms of EIMD have been extensively reviewed $[26,27]$ and contribute to the commonly observed reduction in muscle torque generation and occurrence of delayed onset muscle soreness (DOMS) $[28,29]$, temporarily reducing performance. Thus, WBC's potential ability to minimise EIMD would be an important benefit in its employment during recovery. WBC treatments were shown to enhance recovery of muscle torque compared with a control an hour following muscle damaging exercise [30,31], with benefits lasting up to 4 days [32]. The two former studies examined recovery after eccentric hamstring exercises and a 48-minute simulated trail run respectively, demonstrating lower pain perception. However, some WBC studies have revealed no impact on soreness, utilising eccentric leg extension exercises [23] and 30 metre interval sprints [33]. Whilst not necessarily proving that performance is enhanced, it is likely that athletes experiencing mitigated muscle soreness will feel less pain upon movement during intense training sessions within a short time frame. The choice of exercise protocol may affect the outcome of muscle damage markers more than the choice of cryotherapy protocol.

EIMD can be gauged by creatine kinase (CK), a muscle enzyme which leaks into the circulation following muscle fibre microtears [26]. Studies have revealed that WBC impacts plasma CK $[11,34]$, whereas others have revealed no effect $[31,33]$. Since plasma CK levels are affected by clearance rate from the blood [35] and CK exists in various tissue-specific isoforms, the validity and reliability of plasma CK levels post exercise should be interpreted with caution.

\section{Anti-inflammatory response}

The pronounced inflammatory response post EIMD (i.e. leukocyte infiltration and upregulation of pro-inflammatory 
cytokines) may result in further muscle damage [32]. Thus, a cryotherapy anti-inflammatory effect may be significant for promoting muscle recovery. It has been theorised that extreme cold can attenuate the activity of soluble intercellular adhesion molecule-1 (sICAM-1) [32]. This is an important molecule involved in the recruitment and adhesion of leukocytes to muscle tissue, a key event in the initial inflammatory response [32]. Whilst the evidence for mitigated sICAM following singular WBC treatments is lacking, several studies adopting a variety of WBC protocols have indicated a potential protective effect against post exercise inflammation $[34,36,37]$ with specific markers ranging from increases in the anti-inflammatory cytokine IL-1 receptor antagonist [36], decreases in pro-inflammatory cytokine IL-1 $\beta[36,37]$, and decreased tumor necrosis factor $\alpha$ [34].

\section{Autonomic nervous system and cardiovascular effects}

WBC can significantly impact the autonomic nervous system with concomitant effects on recovery. The potential underlying mechanism is adrenergic activation of receptors in the vascular wall. Pronounced increases in noradrenaline has been observed post-WBC [21], which is reflective of sympathetic $\alpha$-adrenergic fibre activation and consequent arterial and cutaneous vasoconstriction. An increase in heart rate variability has been demonstrated, indicative of enhanced parasympathetic recovery following WBC post exercise [20]. Furthermore, [38] discovered an increased stroke volume combined with reduced heart rate and cardiac output. Such changes indicate more efficient cardiovascular function, albeit temporary, post WBC.

\section{Establishing optimal procedures for WBC treatment - implications for performance}

The optimum protocol for WBC remains unclear in terms of physiological benefits, muscle recovery and sports performance. Treatment timing, duration, temperature, frequency and interindividual factors could all be notable factors. All WBC studies identified in the literature examining recovery post exercise are outlined in Table 1 with specific protocol factors specified.

Table 1: Summary table of studies examining WBC for post-exercise recovery, with varying protocol factors.

\begin{tabular}{|c|c|c|c|c|c|c|c|}
\hline Study & $\begin{array}{l}\text { Sample } \\
\text { characteristics }\end{array}$ & $\begin{array}{l}\text { Exercise } \\
\text { protocol/s }\end{array}$ & $\begin{array}{l}\text { Treatment } \\
\text { duration }\end{array}$ & $\begin{array}{l}\text { Treatment } \\
\text { temperature }\end{array}$ & $\begin{array}{l}\text { Treatment } \\
\text { timing post } \\
\text { exercise }\end{array}$ & $\begin{array}{l}\text { Treatment } \\
\text { frequency }\end{array}$ & $\begin{array}{l}\text { Major } \\
\text { effects/ } \\
\text { findings }\end{array}$ \\
\hline $\begin{array}{l}\text { Hausswirth, } \\
\text { et al. [31] }\end{array}$ & $\begin{array}{l}9 \text { runners, } \\
\text { average age } \\
31.8\end{array}$ & $\begin{array}{l}48 \text {-minute } \\
\text { simulated } \\
\text { trail run on } \\
\text { treadmill } \\
\text { (alternating } \\
\text { gradients) }\end{array}$ & 3 mins & $-110^{\circ} \mathrm{C}$ & $<5$ mins & $\begin{array}{l}3 \text { treatments } \\
\text { on } \\
\text { consecutive } \\
\text { days }\end{array}$ & $\begin{array}{l}\text { +ve effect } \\
\text { on muscle } \\
\text { torque, pain } \\
\text { and wellbeing }\end{array}$ \\
\hline $\begin{array}{l}\text { Fonda \& } \\
\text { Sarabon } \\
{[30]}\end{array}$ & $\begin{array}{l}11 \text { males, } \\
\text { active, } \\
\text { average age } \\
26.9\end{array}$ & $\begin{array}{l}\text { Plyometric } \\
\text { exercises } \\
\text { (drop jumps } \\
\text { and leg curls) }\end{array}$ & 3 mins & $-140^{\circ} \mathrm{C}-185^{\circ} \mathrm{C}$ & 1 hour & $\begin{array}{l}7 \text { treatments } \\
\text { on } \\
\text { consecutive } \\
\text { days }\end{array}$ & $\begin{array}{l}\text { +ve effect } \\
\text { on muscle } \\
\text { torque, jump } \\
\text { power and } \\
\text { pain }\end{array}$ \\
\hline $\begin{array}{l}\text { Ferreira- } \\
\text { Junior, et al. } \\
\text { [32] }\end{array}$ & $\begin{array}{l}12 \text { males, } \\
\text { active, } \\
\text { average age } \\
23.9\end{array}$ & $\begin{array}{l}\text { Concentric } \\
\text { and } \\
\text { eccentric } \\
\text { knee } \\
\text { extensions - } \\
6 \text { sets of } 10 \\
\text { repetitions } \\
\text { for each }\end{array}$ & $\begin{array}{l}3 \text { mins } \\
\left(\mathrm{PBC}^{*}\right)\end{array}$ & $-110^{\circ} \mathrm{C}$ & $<5$ mins & Single & $\begin{array}{l}\text { +ve effect } \\
\text { on muscle } \\
\text { torque }\end{array}$ \\
\hline $\begin{array}{l}\text { Ferreira- } \\
\text { Junior, et al. } \\
\text { [39] }\end{array}$ & $\begin{array}{l}26 \text { males, } \\
\text { active, } \\
\text { average age } \\
20.2\end{array}$ & $\begin{array}{l}\text { Drop jumps } \\
-5 \text { sets of } 20 \\
\text { repetitions }\end{array}$ & $\begin{array}{l}3 \text { mins } \\
\left(\mathrm{PBC}^{*}\right)\end{array}$ & $-110^{\circ} \mathrm{C}$ & 10 mins & Single & $\begin{array}{l}\text { +ve effect } \\
\text { on muscle } \\
\text { torque, } \\
\text { muscle } \\
\text { thickness }\end{array}$ \\
\hline $\begin{array}{l}\text { Costello, et } \\
\text { al. [18] }\end{array}$ & $\begin{array}{l}18 \text { Ps - male } \\
\text { and female }\end{array}$ & $\begin{array}{l}\text { Eccentric } \\
\text { knee } \\
\text { extensions - } \\
20 \text { sets of } 5 \\
\text { reps }\end{array}$ & 3 mins & $-110^{\circ} \mathrm{C}$ & $24 \mathrm{hrs}$ & Single & No +ve effect \\
\hline
\end{tabular}


Citation: Haq A, Ribbans WJ, Baross AW (2018) Enhancing the Physiology and Effectiveness of Whole-Body Cryotherapy Treatment for Sports Recovery by Establishing an Optimum Protocol: A Review of Recent Perspectives. J Phys Med 1(1):41-52

\begin{tabular}{|c|c|c|c|c|c|c|c|}
\hline $\begin{array}{l}\text { Vieira, et al. } \\
\text { [40] }\end{array}$ & $\begin{array}{l}12 \text { males, } \\
\text { average } \\
\text { age } 23.9, \\
\text { resistance } \\
\text { trained }\end{array}$ & $\begin{array}{l}\text { Concentric } \\
\text { and } \\
\text { eccentric } \\
\text { knee } \\
\text { extensions - } \\
6 \text { sets of } 10 \\
\text { repetitions }\end{array}$ & 3 mins & $-110^{\circ} \mathrm{C}$ & $<5$ mins & Single & No +ve effect \\
\hline $\begin{array}{l}\text { Ziemann, et } \\
\text { al. [34] }\end{array}$ & $\begin{array}{l}12 \text { male tennis } \\
\text { players }\end{array}$ & $\begin{array}{l}5 \text { days } \\
\text { moderate } \\
\text { intensity } \\
\text { training - } \\
\text { strength, } \\
\text { endurance, } \\
\text { agility }\end{array}$ & 3 mins & $-120^{\circ} \mathrm{C}$ & $\begin{array}{l}\text { N/A (9:30 } \\
\text { and 17:30 } \\
\text { each day) }\end{array}$ & $\begin{array}{l}2 \text { treatments } \\
\text { a day for } 5 \\
\text { consecutive } \\
\text { days }\end{array}$ & $\begin{array}{l}\text { +ve effects on } \\
\text { inflammation, } \\
\mathrm{CK}, \mathrm{VO}_{2} \text {, heart } \\
\text { rate }\end{array}$ \\
\hline $\begin{array}{l}\text { Wozniak, et } \\
\text { al. [11] }\end{array}$ & $\begin{array}{l}21 \text { kayakers, } \\
\text { male and } \\
\text { female, } \\
\text { average age } \\
24\end{array}$ & $\begin{array}{l}10 \text { days of } \\
\text { training - } \\
\text { endurance, } \\
\text { strength and } \\
\text { water }\end{array}$ & 3 mins & $-120^{\circ} \mathrm{C}-140^{\circ} \mathrm{C}$ & ?? & $\begin{array}{l}3 \text { treatments } \\
\text { a day for } 10 \\
\text { consecutive } \\
\text { days } \\
\text { (trained). } \\
\text { Single } \\
\text { (untrained) }\end{array}$ & $\begin{array}{l}\text { +ve effect on } \\
\text { CK activity } \\
\text { (i.e. reduced } \\
\text { activity) } \\
\text { after } 6 \text { days } \\
\text { training }\end{array}$ \\
\hline $\begin{array}{l}\text { Pournot, et } \\
\text { al. [36] }\end{array}$ & $\begin{array}{l}11 \text { male } \\
\text { runners, } \\
\text { average age } \\
31.8\end{array}$ & $\begin{array}{l}\text { 48-minute } \\
\text { simulated } \\
\text { trail run on } \\
\text { treadmill } \\
\text { (alternating } \\
\text { gradients) }\end{array}$ & 3 mins & $-110^{\circ} \mathrm{C}$ & $<30$ mins & $\begin{array}{l}4 \text { treatments } \\
\text { on } \\
\text { consecutive } \\
\text { days }\end{array}$ & $\begin{array}{l}\text { +ve effect } \\
\text { on cytokines } \\
\text { (i.e. anti- } \\
\text { inflammation) }\end{array}$ \\
\hline $\begin{array}{l}\text { Selfe, et al. } \\
\text { [10] }\end{array}$ & $\begin{array}{l}14 \text { male } \\
\text { rugby players, } \\
\text { average age } \\
24 .\end{array}$ & Rugby fixture & $\begin{array}{l}1,2 \text { or } 3 \\
\text { mins }\end{array}$ & $-135^{\circ} \mathrm{C}$ & $12-24 \mathrm{hrs}$ & & $\begin{array}{l}\text { No effect on } \\
\text { cytokines, +ve } \\
\text { and -ve effect } \\
\text { on tissue } \\
\text { oxygenation. }\end{array}$ \\
\hline $\begin{array}{l}\text { Kruger, et } \\
\text { al. [41] }\end{array}$ & $\begin{array}{l}11 \text { male } \\
\text { athletes, } \\
\text { average age } \\
25.9\end{array}$ & $\begin{array}{l}5 \times 5 \\
\text { minutes } \\
\text { of high } \\
\text { intensity } \\
\text { running }\end{array}$ & 3 mins & $-110^{\circ} \mathrm{C}$ & 45 mins & Single & $\begin{array}{l}\text { +ve effect } \\
\text { on tissue } \\
\text { oxygenation, } \\
\mathrm{VO}_{2} .\end{array}$ \\
\hline $\begin{array}{l}\text { Wozniak, et } \\
\text { al. [15] }\end{array}$ & $\begin{array}{l}20 \text { elite male } \\
\text { kayakers, } 10 \\
\text { untrained } \\
\text { men }\end{array}$ & $\begin{array}{l}\text { Kayak } \\
\text { training, } \\
\text { including } \\
\text { strength and } \\
\text { endurance } \\
\text { training }\end{array}$ & 3 mins & $\begin{array}{l}-120^{\circ} \mathrm{C}-140 \\
{ }^{\circ} \mathrm{C}\end{array}$ & ?? & $\begin{array}{l}3 \text { treatments } \\
\text { a day for } 10 \\
\text { consecutive } \\
\text { days } \\
\text { (trained). } \\
\text { Single } \\
\text { (untrained) }\end{array}$ & $\begin{array}{l}\text { +ve effect on } \\
\text { antioxidants } \\
\text { (e.g. } \\
\text { superoxide } \\
\text { dismutase) }\end{array}$ \\
\hline $\begin{array}{l}\text { Ziemann, et } \\
\text { al. [37] }\end{array}$ & $\begin{array}{l}18 \text { males, } \\
\text { average age } \\
21.7\end{array}$ & $\begin{array}{l}\text { 30-minute } \\
\text { step ups }\end{array}$ & 3 mins & $-110^{\circ} \mathrm{C}$ & $\begin{array}{l}\text { N/A (8:00 } \\
\text { and 16:00 } \\
\text { each day) }\end{array}$ & $\begin{array}{l}2 \text { treatments } \\
\text { a day for } 5 \\
\text { consecutive } \\
\text { days }\end{array}$ & $\begin{array}{l}\text { +ve effect on } \\
\text { inflammation, } \\
\text { CK, DOMS, } \\
\text { cholesterol } \\
\text { (LDL/HDL } \\
\text { ratio) }\end{array}$ \\
\hline
\end{tabular}


Citation: Haq A, Ribbans WJ, Baross AW (2018) Enhancing the Physiology and Effectiveness of Whole-Body Cryotherapy Treatment for Sports Recovery by Establishing an Optimum Protocol: A Review of Recent Perspectives. J Phys Med 1(1):41-52

\begin{tabular}{|c|c|c|c|c|c|c|c|}
\hline $\begin{array}{l}\text { Schaal, et } \\
\text { al. [50] }\end{array}$ & $\begin{array}{l}10 \text { elite female } \\
\text { swimmers, } \\
\text { average age } \\
20.4\end{array}$ & $\begin{array}{l}\text { 2-week } \\
\text { intense } \\
\text { swimming } \\
\text { training }\end{array}$ & 3 mins & $-110^{\circ} \mathrm{C}$ & $\begin{array}{l}\text { N/A } \\
\text { (between } \\
\text { 18:00-19:00 } \\
\text { each day) }\end{array}$ & $\begin{array}{l}14 \\
\text { treatments } \\
\text { on } \\
\text { consecutive } \\
\text { days }\end{array}$ & $\begin{array}{l}\text { +ve effect } \\
\text { on salivary } \\
\alpha \text {-amylase, } \\
\text { blood } \\
\text { lactate, sleep } \\
\text { duration } \\
\text { and sleep } \\
\text { efficiency. }\end{array}$ \\
\hline $\begin{array}{l}\text { Grasso, et } \\
\text { al. [48] }\end{array}$ & $\begin{array}{l}25 \text { male } \\
\text { rugby players, } \\
\text { average age } \\
25.6\end{array}$ & $\begin{array}{l}\text { Rugby } \\
\text { training }\end{array}$ & 3 mins & $-140^{\circ} \mathrm{C}$ & $\begin{array}{l}\text { ?? (morning } \\
\text { and evening) }\end{array}$ & $\begin{array}{l}2 \text { treatments } \\
\text { a day for } 7 \\
\text { consecutive } \\
\text { days }\end{array}$ & $\begin{array}{l}\text { +ve effect on } \\
\text { cortisol and } \\
\text { testosterone }\end{array}$ \\
\hline $\begin{array}{l}\text { Russell, et } \\
\text { al. [33] }\end{array}$ & $\begin{array}{l}14 \text { Premier } \\
\text { League } \\
\text { academy } \\
\text { football } \\
\text { players, } \\
\text { average age } \\
18\end{array}$ & $\begin{array}{l}15 \times 30 \mathrm{~m} \\
\text { sprints }\end{array}$ & 2 mins & $-135^{\circ} \mathrm{C}$ & 20 mins & Single & $\begin{array}{l}\text { +ve effect on } \\
\text { testosterone. } \\
\text { No effect } \\
\text { on other } \\
\text { variables. }\end{array}$ \\
\hline $\begin{array}{l}\text { Abaidia, et } \\
\text { al. [60] }\end{array}$ & $\begin{array}{l}10 \text { males, } \\
\text { active, } \\
\text { average age } \\
23.4\end{array}$ & $\begin{array}{l}\text { Eccentric } \\
\text { knee flexions } \\
\text { - } 5 \text { sets of } 15 \\
\text { repetitions }\end{array}$ & 3 mins & $-110^{\circ} \mathrm{C}$ & 5 mins & Single & $\underset{* *}{\text { No }}$ +ve effect. \\
\hline $\begin{array}{l}\text { Hohenauer, } \\
\text { et al. [61] }\end{array}$ & $\begin{array}{l}10 \text { males, } \\
\text { average age } \\
25.8\end{array}$ & $\begin{array}{l}\text { Drop jumps } \\
\text { - } 5 \text { sets of } 20 \\
\text { repetitions }\end{array}$ & $\begin{array}{l}2 \text { mins } \\
\left(\mathrm{PBC}^{*}\right)\end{array}$ & $-135^{\circ} \mathrm{C}$ & $<10$ mins & Single & $\begin{array}{l}\text { Small +ve } \\
\text { effect on } \\
\text { muscle } \\
\text { torque and } \\
\text { soreness }^{* *}\end{array}$ \\
\hline $\begin{array}{l}\text { Wilson, et } \\
\text { al. [44] }\end{array}$ & $\begin{array}{l}10 \text { male } \\
\text { runners, } \\
\text { average age } \\
37.7\end{array}$ & $\begin{array}{l}\text { Marathon } \\
\text { race }\end{array}$ & 3 mins & $-85^{\circ} \mathrm{C}$ & 15 mins & $\begin{array}{l}\text { Double } \\
\text { (separated by } \\
15 \text { mins) }\end{array}$ & $\begin{array}{l}\text { No +ve effect } \\
\text { on muscle } \\
\text { torque, but } \\
\text { slight +ve } \\
\text { effect on } \\
\text { soreness. }\end{array}$ \\
\hline $\begin{array}{l}\text { Mawhinney, } \\
\text { et al. [24] }\end{array}$ & $\begin{array}{l}10 \text { males, } \\
\text { average age } \\
22.3\end{array}$ & $\begin{array}{l}\text { Steady state } \\
\text { cycling until } \\
\text { core temp } 38 \\
{ }^{\circ} \mathrm{C} \text { reached } \\
\text { (average } 45 \\
\text { mins) }\end{array}$ & 2 mins & $-110^{\circ} \mathrm{C}$ & 10 mins & Single & $\begin{array}{l}\text { Reduction } \\
\text { in muscle } \\
\text { temperature, } \\
\text { heart rate }^{* *}\end{array}$ \\
\hline $\begin{array}{l}\text { Krueger, et } \\
\text { al. [53] }\end{array}$ & $\begin{array}{l}11 \text { male } \\
\text { athletes, } \\
\text { average age } \\
25.9\end{array}$ & $\begin{array}{l}5 \times 5 \\
\text { minutes } \\
\text { of high } \\
\text { intensity } \\
\text { running }\end{array}$ & 3 mins & $-110^{\circ} \mathrm{C}$ & 45 mins & Single & $\begin{array}{l}\text { No }+v e \\
\text { effect on } \\
\text { inflammation, } \\
\text { testosterone }\end{array}$ \\
\hline
\end{tabular}

*PBC - Partial body cryotherapy (head not exposed to cold); ${ }^{* *}$ Studies by Hohenauer, et al. [61] and Mawhinney, et al. [24] only compared WBC effects to CWI, with no control group. Hence, difficult to draw conclusions on efficacy of WBC alone.

\section{Timing}

It would be useful to determine at what stage of recovery post-exercise WBC still evokes beneficial effects, since this has not been addressed adequately in the literature. Most studies examining the positive effect of WBC on muscle recovery applied treatment within 15 minutes post-exercise. For instance, Hausswirth, et al. [31], Ferreira-Junior, et al. [32] and Ferreira-Junior, et al. [39] all reported significant benefits 
Citation: Haq A, Ribbans WJ, Baross AW (2018) Enhancing the Physiology and Effectiveness of Whole-Body Cryotherapy Treatment for Sports Recovery by Establishing an Optimum Protocol: A Review of Recent Perspectives. J Phys Med 1(1):41-52

to muscle torque following WBC treatments within this short time frame. However, such benefits are not consistent in the literature. Vieira, et al. [40] discovered no benefits in muscle recovery when applying WBC immediately after a muscle damaging exercise bout consisting of eccentric and concentric knee extensions. Interestingly, the same exercise and cryotherapy protocol was adopted by Ferreira-Junior, et al. [39], as well as similar sample characteristics (i.e. resistance trained males), making it very difficult to explain the discrepant findings.

Treatments beyond 30 minutes post exercise are less common, but Fonda and Sarabon [30] and Kruger, et al. [41] reported some benefits to recovery using WBC 45 minutes and 1-hour post-exercise respectively. The latter reported that WBC can boost acute muscle recovery and running economy, as indicated by muscle oxygenation and $\mathrm{VO}_{2}$ increasing and decreasing respectively during a repeated same-day treadmill running test to exhaustion. Additionally, in the same study, a large effect size of 0.86 was noted for lower core temperatures in participants who had undergone WBC post-exercise compared to participants undergoing passive recovery. This could have contributed to the performance mismatch between the two groups, especially since a cooler body core can enhance exercise tolerance and delay muscle fatigue [42]. Sports practitioners could use the findings of Kruger, et al. [41] to support performance with WBC treatments applied as late as an hour post exercise, when training or competing in quick succession.

Conversely, Costello, et al. [23] found that applying WBC 24 hours post muscle damaging exercise bout had no beneficial effect on muscle torque or DOMS for up to 3 days. It is conceivable that the 24-hour period after strenuous exercise represents a window when cryotherapy can intervene to influence muscle damage progression. The acute inflammatory response (e.g. leukocyte infiltration) immediately after damaging exercise is believed to contribute to ensuing secondary muscle damage and loss in muscle function over subsequent days [32]. It has been reported that myofibrillar disruption is most prominent 24 hours postdamaging exercise [43], which might explain why treatments after 24 hours are too late to benefit recovery. Thus, there may be a specific time point beyond which cryotherapy cannot be applied to produce optimal recovery. Logistically, it may not be practical to undergo WBC immediately after hard training/competition due to factors such as cool down, transport and treatment accessibility.

To conclude, the timing of cryotherapy treatments may be significant in influencing the efficacy for post exercise recovery. Clear benefits have been discovered following treatments applied as late as one-hour post exercise, but research on the impact of further delayed treatments is lacking. The comparative impact of cryotherapy throughout different stages of the recovery period between 1 and 24 hours post exercise warrants further investigation.

\section{Temperature}

The vast majority of WBC studies utilise temperatures in the range of $-110{ }^{\circ} \mathrm{C}$ to $-140{ }^{\circ} \mathrm{C}$ and there are discrepant findings in recovery responses, with benefits being reported on both ends of the spectrum. For instance, Ferreira-Junior, et al. [32], Ferreira-Junior, et al. [39] and Kruger, et al. [41] discovered clear recovery benefits following single $-110{ }^{\circ} \mathrm{C}$ treatments, whilst Fonda and Sarabon [30] reported some recovery benefits (e.g. jump power) using treatments in the range of $-140{ }^{\circ} \mathrm{C}$ to $-185^{\circ} \mathrm{C}$. It should be noted that the latter study used partial body cryotherapy (similar to WBC except the face is not directly exposed to cold) for 7 treatments on consecutive days. As discussed later, frequency could be a significant factor influencing the efficacy of the treatment, so it would be precarious to draw direct comparisons between this study and those using $-110^{\circ} \mathrm{C}$.

The warmest WBC treatment temperature applied in the literature was $-85^{\circ} \mathrm{C}$ [44]. They interestingly discovered that recovery following a marathon race was detrimental compared to cold water bath treatments and placebo, with regards to muscle torque production and plasma CK. However, WBC appeared to benefit muscle soreness and stress perception compared to the other treatments. It should be noted that the unconventional exercise and cryotherapy protocols (two short bouts of $-85^{\circ} \mathrm{C}$ ) used in this study makes it difficult to draw tenable comparisons and conclusions in the context of the wider literature.

A few studies have reported benefits following treatments in the middle of the $-110{ }^{\circ} \mathrm{C}$ to $-140{ }^{\circ} \mathrm{C}$ range. For instance, Ziemann, et al. [34] and Russell, et al. [33] used $-120^{\circ} \mathrm{C}$ and $-135{ }^{\circ} \mathrm{C}$ respectively, whereby reduced inflammation and increased testosterone was observed respectively. Due to the superior thermal gradient and potential for heat exchange, it would be reasonable to hypothesise that more extreme cold temperatures would more profoundly affect physiological responses, such as reductions in skin and muscle temperatures. It is acknowledged that there have been no studies assessing the effect of manipulating WBC treatment temperatures alone, making it difficult to draw definitive conclusions on the influence of this factor for sports recovery. Additionally, it is probable that there are discrepancies in the reported temperatures of WBC chambers and actual temperatures whilst the subjects undergo their treatments, as indicated in the review by Bouzigon, et al. [13]. Whilst small fluctuations in chamber temperature during treatments are inevitable, this would also make it difficult to conclude the precise impact of treatment temperature manipulations on physiology and performance outcomes.

To summarise, due to the short duration of treatments, it is unlikely that temperature plays a significant role in influencing the efficacy of WBC for post exercise recovery. Future studies and treatments would be sensible to stay within the $-110{ }^{\circ} \mathrm{C}$ to $-140{ }^{\circ} \mathrm{C}$ range.

\section{Duration}

It has been suggested that WBC durations exceeding 2.5 minutes may not be necessary due to added thermal discomfort [45]. Selfe, et al. [10] attempted to identify the optimum treatment duration by comparing three different WBC durations $\left(-135^{\circ} \mathrm{C}\right)$ as recovery interventions following rugby matches. They concluded that a 2-minute exposure 
Citation: Haq A, Ribbans WJ, Baross AW (2018) Enhancing the Physiology and Effectiveness of Whole-Body Cryotherapy Treatment for Sports Recovery by Establishing an Optimum Protocol: A Review of Recent Perspectives. J Phys Med 1(1):41-52

may be ideal taking into account several physiological variables. The rationale for proposing a 2-minute exposure as opposed to 3 minutes is questionable on the premise that skin temperatures were significantly lower immediately post 3-minute cryotherapy than 2 minutes, whilst other variables (e.g. tissue oxygenation, thermal comfort), were not significantly different between these durations. The longest treatment duration identified in the literature is 3 minutes, 40 seconds [23], which as mentioned prior, noted pronounced reductions in skin and deep muscle tissue temperature. However, such reductions have been matched or exceeded in subsequent studies utilising shorter durations [20,24]. Durations of longer than 4 minutes are not advised due to safety concerns, which have been highlighted previously $[13,46]$ and are well established. Similar to temperature, it is difficult to make conclusive statements regarding the impact of treatment duration, since the vast majority of WBC studies have used treatments lasting 3 minutes. Whether subtle differences in treatment duration have a significant impact on physiology and performance is contentious.

\section{Frequency}

Studies indicate that superior effects are acquired by performing multiple WBC sessions in succession $[19,47]$. Every study identified in Table 1 that used multiple WBC treatments discovered some significant positive effect. An accumulative physiological effect following multiple sessions seems plausible. Higher treatment frequencies can augment anti-inflammatory effects [34] and the testosterone to cortisol ratio [48], the latter of which can impact protein synthesis and muscle glycogen replenishment [49].

Ziemann, et al. [34] demonstrated positive recovery effects and a potential link between physiology and performance by applying repetitive cryotherapy treatments over 5 days to tennis players. There was reduced inflammation, oxygen uptake and heart rate, as well as improved stroke effectiveness compared to a control group during a subsequent tennis drill.

One notable study that applied frequent WBC interventions (every day throughout a 2-week training programme) noted significant benefits on sleep quality, promoting recovery and reducing fatigue in the run up to competitions [50]. The mechanism for improved sleep is not clear, however an enhanced parasympathetic activation is plausible. There is scope for further development in this area since the impact on sports performance could be considerable.

Conversely, the possibility of repetitive WBC bouts having a negative impact on long term training adaptations should not be discounted. It has been reported that following a multiweek training protocol, increases in blood flow and arterial vessel diameters were less evident for repetitively cooled limbs via CWI than non-cooled limbs post-exercise [51], indicative of attenuated adaptations. Furthermore, Roberts, et al. [52] demonstrated that repetitive $\mathrm{CWI}$ application over several weeks attenuated anabolic signalling mechanisms following a resistance training programme, blunting muscle strength and mass development. They proposed that the reduced muscle blood flow post-cryotherapy reduces amino acid delivery, consequently reducing activation of signalling pathways.
As mentioned, prior, muscle damaging exercise bouts causes a pronounced inflammatory response characterised by infiltration of leukocytes into muscle tissue and consequent upregulation of pro-inflammatory cytokines, which act in concert to degrade muscle tissue further, thus amplifying the initial damage [27]. It is worth considering that this initial extensive inflammatory response is integral to muscular repair and regeneration [27], thus the potential extent to which repetitive WBC treatments might curtail the post-exercise inflammatory response should not be overlooked. The WBCinduced mitigation of EIMD/inflammation could be counterproductive for adaptive changes in the long term. This can have implications for the application of WBC treatments in relation to periodisation - i.e. it may be preferable to use WBC when rapid recovery is needed in the midst of a competitive phase as opposed to during pre-season where adaptations may be prioritised. On the contrary, a recent study revealed that WBC did not alter inflammatory markers following a high intensity exercise bout [53]. Due to the lack of clarity in the literature, it would be beneficial to investigate the potential impact of repetitive WBC treatments on long term training adaptations.

In conclusion, it seems reasonable to assume that more frequent WBC treatments within a short time frame lead to better results for post exercise recovery. One should consider the potential knock-on effect on chronic adaptations and of course, the cost implications of applying frequent WBC treatments for superior impact.

\section{Inter-individual factors}

The exact optimal WBC regime can largely depend upon inter-individual factors. Studies have shown that adipose tissue content is inversely related to the degree of muscle tissue cooling owing to its insulating properties [54,55]. This insulation, due to reduced conductive heat transfer from the body core to the external surrounding [22], can reduce the overall effect of cold exposure. Since greater body insulation reduces the magnitude of muscle cooling, it is conceivable that higher body fat individuals would retain internal core and tissue temperatures to larger extents following cryotherapy, compared to leaner individuals. This could jeopardise their ability to recover from exercise. Athletes' body compositions should therefore be considered before applying cryotherapy treatments. The age of WBC users could be an additional factor. A reduced responsiveness of ageing blood vessels [56] may be significant because of the potential impact on blood redistribution and heat transfer. Indeed, adipose tissue content naturally increases with age due to a reduction in basal metabolic rate [57].

Research on sex differences in cryotherapy responses is generally limited. Two studies have demonstrated that females can experience more pronounced skin temperature reductions than males following single WBC treatments $[58,59]$ which could be explained by higher body fat contents since a significant correlation was noted between adiposity and skin temperature. However, no studies to date have examined sex differences in WBC responses for recovery post exercise. Only one study identified in Table 1 tested a sample 
Citation: Haq A, Ribbans WJ, Baross AW (2018) Enhancing the Physiology and Effectiveness of Whole-Body Cryotherapy Treatment for Sports Recovery by Establishing an Optimum Protocol: A Review of Recent Perspectives. J Phys Med 1(1):41-52

of entirely female participants and reported significant recovery benefits, such as sleep quality and reduced blood lactate [50]. Future research examining similar parameters in male participants might further our understanding of the influence of sex on the effectiveness of WBC treatment.

The research would therefore suggest that interindividual factors such as body fat, age and sex could have a significant role influencing the efficacy of WBC treatment due to implications on physiological responses. Nonetheless, further research involving causal-comparative parallel groups designs are necessary for establishing better conclusions on this matter. The potential association between interindividual variabilities in EIMD mechanisms and response to cryotherapy treatments is an issue that has not been addressed fully in the literature.

\section{Conclusions}

Whole body cryotherapy is an emerging method of sports recovery with numerous physiological and performance effects. Some of the benefits that WBC can elicit are due to its proven anti-inflammatory properties and possible impact on other recovery factors, including sleep. Several WBC protocol variables may influence its efficacy. The timing of treatments post-exercise may particularly impact effectiveness and the inter-individual variability (e.g. body fat content and age) of responses to WBC should not be ignored. Whilst concluding an optimal protocol for WBC remains a challenge, we propose that 2.5 -minute treatments at between $-110{ }^{\circ} \mathrm{C}$ and -140 ${ }^{\circ} \mathrm{C}$ taken within 60 minutes post exercise may induce the most desirable results regarding muscle damage recovery, particularly for young males with low body fat contents. There is a lack of evidence to support a relationship between severity of WBC treatment (temperature and duration) and physiological and performance parameters (e.g. skin temperatures, muscle damage markers), yet a dose-response relationship may be somewhat apparent with regards to the frequency of WBC treatment. The question of whether long term repetitive WBC treatments can hinder adaptations to training is one that warrants further investigation. The overall effectiveness of WBC treatment regarding physiology and performance is likely due to an interplay of contributing factors, although the clarification of an optimised WBC protocol would represent a significant step for athletes and coaches constantly striving for performance advantages.

\section{Acknowledgements}

Tony Kay, Professor of Biomechanics at the University of Northampton, is acknowledged for his insight regarding the content and layout of this review article, as well as reviewing the article before submission.

\section{Conflict of Interest}

The authors report no conflicts of interest.

\section{References}

1. Barnett A (2006) Using recovery modalities between training sessions in elite athletes: does it help? Sports Med 36: 781-796.

2. Dupuy O, Douzi W, Theurot D, et al. (2018). An evidence-based approach for choosing post-exercise recovery techniques to reduce markers of muscle damage, soreness, fatigue, and inflammation: A systematic review with meta-analysis. Front Physiol 9: 403.

3. Halson SL (2013) Recovery techniques for athletes. Sports Sci Exch 26: 1-6.

4. Ihsan M, Watson G, Abbiss CR (2016) What are the physiological mechanisms for post-exercise cold water immersion in the recovery from prolonged endurance and intermittent exercise? Sports Med 46: 1095-1109.

5. Leeder J, Gissane C, van Someren K, et al. (2012) Cold water immersion and recovery from strenuous exercise: a metaanalysis. Br J Sports Med 46: 233-240.

6. Yamauchi T, Kim S, Nogami S, et al. (1981) Extreme cold treatment $\left(-150^{\circ} \mathrm{C}\right)$ on the whole body in rheumatoid arthritis. Rev Rhum 48: 1054.

7. Stanek A, Sieroń A, Cieślar G, et al. (2005) The impact of wholebody cryotherapy on parameters of spinal mobility in patients with ankylosing spondylitis. Ortop Traumatol Rehabil 7: 549-554.

8. Ma SY, Je HD, Jeong JH, et al. (2013) Effects of whole-body cryotherapy in the management of adhesive capsulitis of the shoulder. Arch Phys Med Rehabil 94: 9-16.

9. Bettoni L, Bonomi FG, Zani V, et al. (2013) Effects of 15 consecutive cryotherapy sessions on the clinical output of fibromyalgic patients. Clinical Rheumatology 32: 1337-1345.

10. Selfe J, Alexander J, Costello JT, et al. (2014) The effect of three different $\left(-135^{\circ} \mathrm{C}\right)$ whole body cryotherapy exposure durations on elite rugby league players. Plos One 9: e86420.

11. Wozniak A, Wozniak B, Drewa G, et al. (2007) The effect of whole body cryostimulation on lysosomal enzyme activity in kayakers during training. Eur J Appl Physiol 100: 137-142.

12. Banfi G, Lombardi G, Colombini A, et al. (2009) Whole-body cryotherapy in athletes. Sports Med 40: 509-517.

13. Bouzigon R, Grappe F, Ravier G, et al. (2016) Whole and partialbody cryostimulation/cryotherapy: current technologies and practical applications. J Thermal Biology 61: 67-81.

14. Rose C, Edwards KM, Siegler J, et al. (2017) Whole-body cryotherapy as a recovery technique after exercise: A review of the literature. Int J Sports Med 38: 1049-1060.

15. Wozniak A, Wozniak B, Drewa G, et al. (2007) The effect of wholebody cryostimulation on the prooxidant-antioxidant balance in blood of elite kayakers after training. Eur J Appl Physiol 101: 533537.

16. Young AJ, Sawka MN, Pandolf KB (1996) Physiology of cold exposure. Nutritional needs in cold and high-altitude environments: applications for military personnel in field operations. The National Academies Press. 127-148.

17. White GE, Wells GD (2013) Cold-water immersion and other forms of cryotherapy: physiological changes potentially affecting recovery from high-intensity exercise. Extrem Physiol Med 2: 26.

18. Costello JT, Algar LA, Donnelly AE (2012) Effects of whole-body cryotherapy $\left(-110^{\circ} \mathrm{C}\right)$ on proprioception and indices of muscle damage. Scand J Med Sci Sports 22: 90-98.

19. Lubkowska A, Dołęgowska B, Szyguła Z (2012) Whole-Body cryostimulation - potential beneficial treatment for improving antioxidant capacity in healthy men - significance of the number of sessions. Plos One 7: e46352. 
Citation: Haq A, Ribbans WJ, Baross AW (2018) Enhancing the Physiology and Effectiveness of Whole-Body Cryotherapy Treatment for Sports Recovery by Establishing an Optimum Protocol: A Review of Recent Perspectives. J Phys Med 1(1):41-52

20. Hausswirth C, Schaal K, Le Meur Y, et al. (2013) Parasympathetic activity and blood catecholamine responses following a single partial-body cryostimulation and a whole-body cryostimulation. PLoS One. 8: e72658.

21. Leppäluoto J, Westerlund T, Huttunen P, et al. (2008) Effects of long-term whole-body cold exposures on plasma concentrations of ACTH, beta-endorphin, cortisol, catecholamines and cytokines in healthy females. Scand J Clin Lab Invest 68: 145-153.

22. Bleakley CM, Davison, Gareth W (2010) Cryotherapy and inflammation: evidence beyond the cardinal signs. Physical Therapy Reviews 15: 430-435

23. Costello JT, Culligan K, Selfe S (2012) Muscle, skin and core temperature after $-110^{\circ} \mathrm{C}$ cold air and $8^{\circ} \mathrm{C}$ water treatment. Plos One 7.

24. Mawhinney C, Low DA, Jones H, et al. (2017) Cold-water mediates greater reductions in limb blood flow than whole body cryotherapy. Medicine and Science in Sports and Exercise 49: 1252-1260.

25. Howatson G, van Someren KA (2008) The prevention and treatment of exercise-induced muscle damage. Sports Med 38 : 483-503.

26. Clarkson PM, Hubal MJ (2002) Exercise-induced muscle damage in humans. Am J Phys Med Rehabil 81: S52-S69.

27. Peake JM, Neubauer O, Della Gatta PA, et al. (2017) Muscle damage and inflammation during recovery from exercise. J App Physiol 122: 559-570.

28. Bailey DM, Erith SJ, Griffin PJ, et al. (2007) Influence of cold-water immersion on indices of muscle damage following prolonged intermittent shuttle running. J Sports Sciences 25: 1163-1170.

29. Dolci A, Fortes MB, Walker FS, et al. (2015) Repeated muscle damage blunts the increase in heat strain during subsequent exercise heat stress. Eur J Appl Physiol 115: 1577-1588.

30. Fonda B, Sarabon N (2013) Effects of whole-body cryotherapy on recovery after hamstring damaging exercise: A crossover study. Scand J Med Sci Sports 23: e270-e278.

31. Hausswirth C, Louis J, Bieuzen F, et al. (2011) Effects of wholebody cryotherapy vs. far-infrared vs. passive modalities on recovery from exercise-induced muscle damage in highly-trained runners. Plos One 6: e27749.

32. Ferreira-Junior JB, Bottaro M, Vieira CA, et al. (2014) Effects of partial-body cryotherapy $\left(-110^{\circ} \mathrm{C}\right)$ on muscle recovery between high-intensity exercise bouts. Int J Sports Med 35: 1155-1160.

33. Russell M, Birch J, Love T, et al. (2016) The effects of a single whole-body cryotherapy exposure on physiological, performance and perceptual responses of professional academy soccer players following repeated sprint exercise. J Strength Cond Res 31: 415-421.

34. Ziemann E, Olek RA, Kujach S, et al. (2012) Five-day whole-body cryostimulation, blood inflammatory markers, and performance in high-ranking professional tennis players. J Athl Train 47: 664-672.

35. Brancaccio $P$, Maffulli N, Limongelli FM (2007) Creatine kinase monitoring in sport medicine. Br Med Bull 81-82: 209-230.

36. Pournot H, Bieuzen F, Louis J, et al. (2011) Time-course of changes in inflammatory response after whole-body cryotherapy multi exposures following severe exercise. Plos One 6: 22748.

37. Ziemann E, Olek RA, Grzywacz T, et al. (2013) Whole-body cryostimulation as an effective way of reducing exercise-induced inflammation and blood cholesterol in young men. Eur Cytokine Netw 25: 14-23.

38. Zalewski P, Klawe JJ, Pawlak J, et al. (2013) Thermal and hemodynamic response to whole-body cryostimulation in healthy subjects. Cryobiology 66: 295-302.

39. Ferreira-Junior JB, Bottaro M, Vieira A, et al. (2015) One session of partial-body cryotherapy $\left(-110^{\circ} \mathrm{C}\right)$ improves muscle damage recovery. Scand J Med Sci Sports 25: 524-530.

40. Vieira A, Bottaro M, Ferreira-Junior JB, et al. (2015) Does wholebody cryotherapy improve vertical jump recovery following a high-intensity exercise bout? Open Access J Sports Med 6: 49-54.

41. Krüger M, de Mareés $M$, Dittmar KH, et al. (2015) Whole-body cryotherapy's enhancement of acute recovery of running performance in well-trained athletes. Int J Sports Physiol Perform 10: 605-612.

42. Marino FE (2002) Methods, advantages, and limitations of body cooling for exercise performance. Br J Sports Med 36: 89-94.

43. Paulsen G, Mikkelsen UR, Raastad T, et al. (2012) Leucocytes, cytokines and satellite cells: what role do they play in muscle damage and regeneration following eccentric exercise? Exerc Immunol Rev 18: 42-97.

44. Wilson L, Cockburn E, Paice K, et al. (2017) Recovery following a marathon: a comparison of cold water immersion, whole body cryotherapy and a placebo control. Eur J Appl Physiol 118: 153163.

45. Fonda B, De Nardi M, Sarabon N (2014) Effects of whole-body cryotherapy duration on thermal and cardio-vascular response J Therm Biol 42: 52-55.

46. Bleakley CM, Bieuzen F, Davison GW, et al. (2014) Whole-body cryotherapy: empirical evidence and theoretical perspectives. Open Access J Sports Med 10: 25-36.

47. Lubkowska A, Szyguła Z, Chlubek D, et al. (2011) The effect of prolonged whole-body cryostimulation treatment with different amounts of sessions on chosen pro- and anti-inflammatory cytokines levels in healthy men. Scand J Clin Lab Invest 71: 419425.

48. Grasso D, Lanteri P, Di Bernardo C, et al. (2014) Salivary steroid hormone response to whole-body cryotherapy in elite rugby players. J Biol Regul Homeost Agents 28: 291-300.

49. Urhausen A, Gabriel H, Kindermann W (1995) Blood hormones as markers of training stress and overtraining. Sports Med 20: 251-276.

50. Schaal K, LE Meur Y, Louis J, et al. (2015) Whole-body cryostimulation limits overreaching in elite synchronized swimmers. Med Sci Sports Exerc 47: 1416-1425.

51. Yamane $M$, Teruya $H$, Nakano $M$, et al. (2006) Post-exercise leg and forearm flexor muscle cooling in humans attenuates endurance and resistance training effects on muscle performance and on circulatory adaptation. Eur J Appl Physiol 96: 572-580.

52. Roberts LA, Raastad T, Markworth JF, et al. (2015) Post exercise cold water immersion attenuates acute anabolic signalling and long-term adaptations in muscle to strength training. J Physiology 593: 4285-4301.

53. Krueger M, Costello JT, Achtzehn S, et al. (2018) Whole-body cryotherapy $\left(-110^{\circ} \mathrm{C}\right)$ following high-intensity intermittent exercise does not alter hormonal, inflammatory or muscle damage biomarkers in trained males. Cytokine 30310-30317. 
Citation: Haq A, Ribbans WJ, Baross AW (2018) Enhancing the Physiology and Effectiveness of Whole-Body Cryotherapy Treatment for Sports Recovery by Establishing an Optimum Protocol: A Review of Recent Perspectives. J Phys Med 1(1):41-52

54. Myrer WJ, Myrer KA, Measom GJ, et al. (2001) Muscle temperature is affected by overlying adipose when cryotherapy is administered. J Athletic Training 36: 32-36.

55. Otte JW, Merrick MA, Ingersoll CD, et al. (2002) Subcutaneous adipose tissue thickness alters cooling time during cryotherapy. Arch Phys Med Rehabil 83: 1501-1505.

56. Knight J, Nigam Y (2008). Exploring the anatomy and physiology of ageing. Part 1--The cardiovascular system. Nurs Times 104: 26-27.

57. Kenney WL, Wilmore J, Costill D (2011) Physiology of Sport and Exercise. (5 $5^{\text {th }}$ edn), Human Kinetics, UK.

58. Hammond LE, Cuttell S, Nunley P, et al. (2014) Anthropometric characteristics and sex influence magnitude of skin cooling following exposure to whole body cryotherapy. Biomed Res Int 2014: 628-724.

59. Cuttell S, Hammond L, Langdon D, et al. (2017) Individualising the exposure of $-110^{\circ} \mathrm{C}$ whole body cryotherapy: The effects of sex and body composition. J Therm Biol 65: 41-47.

60. Abaïdia AE, Lamblin J, Delecroix B, et al. (2017) Recovery from Exercise-Induced Muscle Damage: Cold-Water Immersion Versus Whole-Body Cryotherapy. I Int J Sports Physiol Perform 12: 402-409.

61. Hohenauer E, Costello JT, Stoop R, et al. (2017) Cold-water or partial-body cryotherapy? Comparison of physiological responses and recovery following muscle damage. Scand J Med Sci Sports 28: 1252-1262. 